\title{
PAPER
}

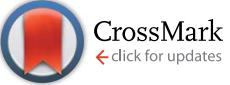

Cite this: J. Mater. Chem. A, 2015, 3, 10935

Received 30th January 2015

Accepted 13th April 2015

DOI: $10.1039 / c 5 t a 00784 d$

www.rsc.org/MaterialsA

\section{Ultrafast charge transfer in solid-state films of pristine cyanine borate and blends with fullerene $\uparrow$}

\begin{abstract}
Jelissa De Jonghe-Risse, ${ }^{a}$ Jakob Heier, ${ }^{b}$ Frank Nüesch ${ }^{\text {bc }}$ and Jacques-E. Moser ${ }^{\star a}$
Photoinduced electron transfer in light-absorbing materials is the first step towards charge separation and extraction in small molecule-based organic solar cells. The excited state dynamics of the cyanine dye cation Cy3 paired with a tetraphenylborate counter-anion (Cy3-B) was studied in pristine solid-state films of the dye and in blends with the electron acceptor material PCBM. Here we show that photoexcited Cy3-B in pure films undergoes intra-ion pair reductive quenching on the picosecond time scale, while in blends with PCBM sub-picosecond formation of the Cy3 oxidized species is observed upon electron injection from the dye excited state into the fullerene. Kinetic competition between light-induced electron- and hole transfer processes strongly depends on the PCBM content in the blends. A high PCBM loading produces a fully intermixed phase, where the cyanine oxidized states appear on ultrashort ( $<160 \mathrm{fs}$ ) time scales. Lower PCBM contents, in contrast, lead to a Cy3-B segregated phase on top of the intermixed phase and slower excited state quenching. These findings show that the phase morphology indeed controls to a large extent the efficiency of primary photoinduced charge separation, on which small molecule-based organic photovoltaic cells rely.
\end{abstract}

\section{Introduction}

Continuous progress in small molecule organic solar cell technology has led lately to promising power conversion efficiencies. ${ }^{1-3}$ Efforts are currently directed towards achieving improved stability and device performances. The increasing interest in these systems and the need for better knowledge of the initial charge generation have led researchers to thoroughly investigate primary ultrafast photoinduced processes., ${ }^{\mathbf{4} 5}$ Solar cells built from cyanine dyes and a fullerene electron acceptor are particularly interesting. Indeed, spontaneous phase demixing in blends of the two materials forms bulk heterojunctions characterized by a rich variety of phase morphologies. ${ }^{6}$ On the other hand, bilayer solar cells based on the cyanine dye Cy3 (1,1'-diethyl-3,3,3',3'-tetramethylcarbocyanine, see the structure in Fig. 1), in particular, have shown a competitive photovoltaic power conversion efficiency, reaching up to $3.7 \% .^{7-12}$ Cyanines are positively charged polymethine dyes and are paired with a negatively charged counter-ion. Anion migration under applied electric fields in solid state

${ }^{a}$ Photochemical Dynamics Group, Institute of Chemical Sciences and Engineering, École Polytechnique Fédérale de Lausanne, CH-1015 Lausanne, Switzerland. E-mail: je.moser@epfl.ch

${ }^{b}$ Laboratory for Functional Polymers, Swiss Federal Laboratories for Materials Science and Technology, EMPA, CH-8600 Dübendorf, Switzerland

'Institute of Materials, École Polytechnique Fédérale de Lausanne, CH-1015 Lausanne, Switzerland

$\uparrow$ Electronic supplementary information (ESI) available. See DOI: $10.1039 / \mathrm{c} 5$ ta00784d cyanine layers has been observed and could be detrimental to small molecule solar cell applications, as the charges building up at the electrode interface would hinder charge extraction. ${ }^{\mathbf{6}, 13}$ In order to restrict ion migration, larger counter-anions can be used such as borate and TRISPHAT. ${ }^{\mathbf{1 4}}$ Their redox photoactivity has nonetheless to be controlled in order to avoid irreversible

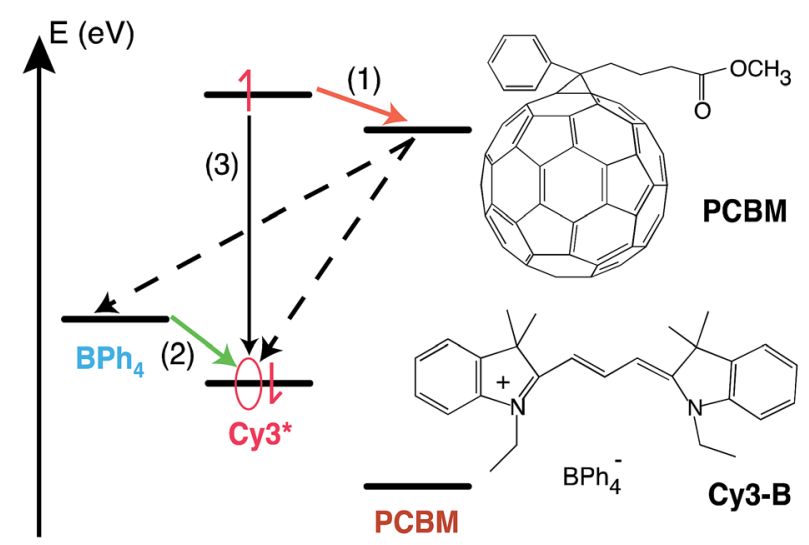

Fig. 1 Schematic layout of energy levels and electron transfer processes following Cy3 photoexcitation in pristine Cy3-B and blends with PCBM. (1) Electron transfer from Cy3 LUMO to PCBM; (2) reductive quenching of the $\mathrm{Cy}_{3}{ }^{*}$ excited state by the tetraphenylborate counter-anion ( $e^{-}$transfer from $\mathrm{BPh}_{4}$ to the $\mathrm{Cy} 3 \mathrm{HOMO}$ ); (3) deactivation of $\mathrm{Cy} 3 *$ through radiative and non-radiative pathways. Back-electron transfer and charge recombination at tetraphenylborate|PCBM junctions appear as dotted lines. The molecular structures of the investigated materials are shown on the right. 
reactions. The photophysical properties of cyanine dyes have been extensively studied in solution ${ }^{15-17}$ and in pristine solidstate films. ${ }^{\mathbf{1 8 - 2 0}} \mathrm{Cy} 3$ excited state dynamics in solution are strongly dependent on the nature of the counter-anion..$^{21-25} \mathrm{Cy} 3$ hexafluorophosphate (Cy3-P) tends to photoisomerize to the cisisomer and has a rather poor fluorescence quantum yield. The excited state decays in a few hundreds of picoseconds, while the cis-isomer absorbing at $580 \mathrm{~nm}$ is characterized by a microsecond lifetime. With the iodide counter-anion, no singlet excited state is observed on the ps time scale, but rather a triplet excited state with nanosecond lifetime absorbing at $620 \mathrm{~nm}$ is formed due to the heavy-atom effect of iodine, enhancing intersystem crossing. In the case of borate counter-anions, it has been shown that electron transfer to the excited state of cyanine (reductive quenching) leads to a reduced dye species $\left(\mathrm{Cy}^{-}\right)$, whose absorption is centered at a wavelength of $430 \mathrm{~nm}$. Alkyl-substituted borates undergo a carbon-boron bond cleavage upon oxidation, generally resulting in unstable product radicals. However, in the case of tetraphenylborate, which is a good electron donor, ${ }^{26,27}$ back-electron transfer from the reduced cyanine species is apparently sufficiently fast to regenerate the dye borate and prevent its decomposition.

The photophysical processes for the cyanine tetraphenylborate salt that undergoes intra ion-pair electron transfer under light irradiation are shown in Fig. 1 (process 2). By adding an electron acceptor such as the fullerene derivative [6,6]-phenyl $\mathrm{C}_{61}$-butyric acid methyl ester (PCBM), kinetic competition between electron transfer to PCBM (process 1) and reductive quenching by tetraphenylborate (process 2) is expected.

$$
\begin{gathered}
\mathrm{Cy}^{*}+\mathrm{PCBM} \rightarrow \mathrm{Cy}^{+}+\mathrm{PCBM}^{-} \\
\mathrm{Cy}^{*}+\mathrm{BPh}_{4} \rightarrow \mathrm{Cy}^{-}+\mathrm{BPh}^{+}
\end{gathered}
$$

In this work we report transient absorption studies on solidstate films of pristine cyanine tetraphenylborate (Cy3-B) and of various blends with PCBM. Kinetic competition between the reductive quenching by tetraphenylborate of the photoexcited Cy3 (eqn (2)) and its oxidative quenching by PCBM (eqn (1)) was monitored. Different PCBM:Сy3-B blend ratios enabled us to alter the yield of the oxidized cyanine $\left(\mathrm{Cy}^{+}\right)$, indicating that intermixed phases are crucial for photoinduced charge separation.

\section{Results and discussion}

Femtosecond pump-probe experiments were carried out on various film compositions. As the photoabsorption of different species overlaps in the visible spectral range, a series of blends was investigated, whose compositions were varied from pure Cy3-B up to a PCBM:Cy3-B molar ratio of 15.5 : 1 . The normalized absorption spectra of thin films of pristine Cy3-B and of bulk heterojunctions formed in blends with PCBM are shown in Fig. 2. The pristine Cy3-B ground state absorption spectrum in solid films extends over wavelengths from approximately 450 to $600 \mathrm{~nm}$ and displays the dye's monomer absorption maximum at $570 \mathrm{~nm}$. This spectrum is similar to that of the Cy3-P cyanine

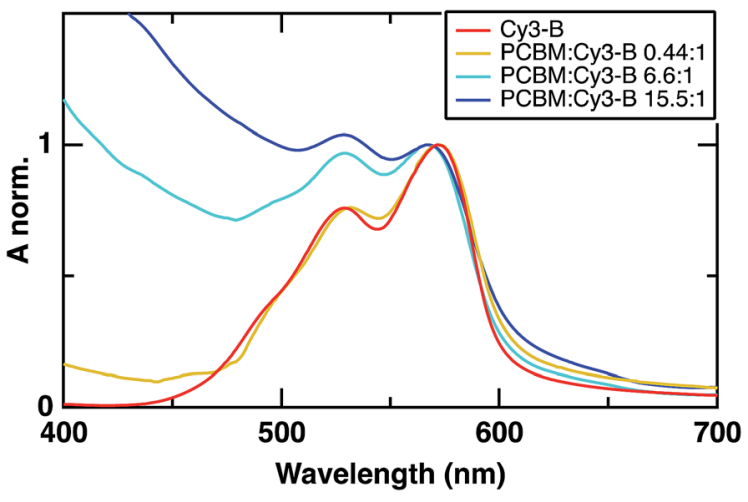

Fig. 2 Normalized steady-state absorption spectra of the pristine Cy3-B film and the various blends with PCBM.

dye with $\mathrm{PF}_{6}{ }^{-}$as a counter-anion. ${ }^{8}$ It is red-shifted and broadened compared to the absorption spectrum measured in solution. Blue shifted features are attributed to dimer and $\mathrm{H}$-aggregate absorption (shoulder around $500 \mathrm{~nm}$ ). Remarkably, the amount of aggregated dye is rather small and does not seem to increase in the presence of PCBM. Absorption of PCBM is quite weak in the visible but increases in the UV region, causing a slight blue shift of the apparent Cy3-B absorption maximum in blends.

\section{Pristine Cy3-B}

For clarity of the discussion, transient absorption results for pristine Cy3-B are presented first. Upon pulsed laser excitation, the appearance of a large negative band located at 550-680 nm is observed instantaneously (Fig. 3a) and is attributed to the Cy3 ground-state bleaching (GSB) due to the similarity to steady-

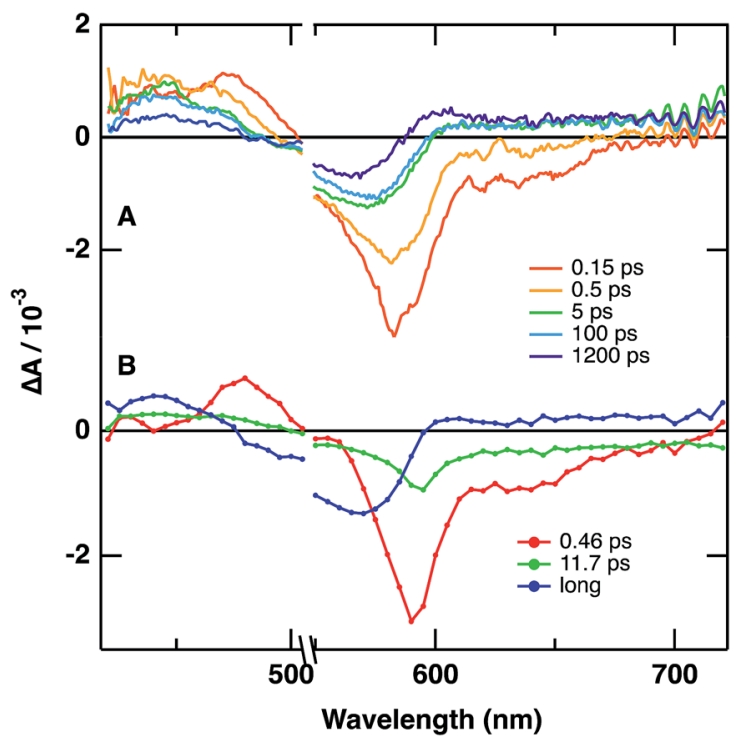

Fig. 3 (a) Transient absorption spectra at various time delays after 530 $\mathrm{nm}$ excitation for pristine Cy3-B and (b) amplitude spectra associated with the time constants resulting from the multiexponential global analysis for pristine Cy3-B. 
state absorbance (Fig. 2). At early time delays, the negative maximum at $580 \mathrm{~nm}$ contains contributions of both the GSB and a weak stimulated emission signal (SE). As the SE vanishes, this maximum shifts to the blue side to $570 \mathrm{~nm}$. The positive transient absorption band observed at 420-500 $\mathrm{nm}$ during the first tenths of picoseconds is assigned to the singlet excited state absorption (ESA), which was already reported by Chatterjee et al. for time-resolved flash photolysis studies on Cy3-P. ${ }^{24,28}$ This photoabsorption compensates the GSB below $550 \mathrm{~nm}$. The fast quenching of the cyanine ESA by the tetraphenylborate counter-anion leads to the formation of the reduced cyanine species $\left(\mathrm{Cy}^{-}\right)$absorbing at about $420-470 \mathrm{~nm}$ and oxidized tetraphenylborate (eqn (2)). This reductive quenching reaction has been observed in solution. ${ }^{25}$ In thin solid films, red shift and broadening of the absorption spectra compared to the same species in solution are commonly observed for molecules, which explains a maximum absorption peak for the $\mathrm{Cy}^{-}$ species located at $420-470 \mathrm{~nm}$ in the pristine film compared to $430 \mathrm{~nm}$ in solution.

Kinetic data were recorded with pulsed laser excitation energy fluence at the sample of $45 \mu \mathrm{J} \mathrm{cm}^{-2}$. No effect of the light intensity was observed, as evidenced by identical dynamics obtained upon application of lower fluences (Fig. S1a $\dagger$ ). The associated spectra resulting from a multiexponential global analysis are shown in Fig. 3b. They bring clear evidence for the reductive quenching mechanism. More details about the procedure applied for the global analysis of multiwavelength kinetic data are provided in the ESI. $\uparrow$ The decay of the red part of the ESA between 460 and $500 \mathrm{~nm}$ is associated with a $0.46 \mathrm{ps}$ time constant and is mirrored by the decay of the SE around 590-680 nm. As the GSB does not decay on this time scale, it is reasonable to assume that the singlet excited state does not fully return to the ground state, and this will be confirmed later. Looking more specifically at the positive transient feature located at 420-460 $\mathrm{nm}$ and assigned to both the ESA and Cy3absorption, it is clear that the ESA is converted into the reduced cyanine species transient signal with a time constant of $0.46 \mathrm{ps}$. The reductive quenching of the excited state by the tetraphenylborate counter-anion is evidenced by the isosbestic point located at $460 \mathrm{~nm}$ (Fig. 3b) between the red part of the ESA and the absorption of the reduced cyanine. The latter signal grows with an 11.7 ps time constant and displays a lifetime that is longer than the $1 \mathrm{~ns}$ maximum optical time delay available with our femtosecond laser setup. Application of nanosecond flash photolysis measurements to identical samples (Fig. 4) confirmed the formation of long-lived reduced cyanine species, whose decay by back electron transfer takes place in the microsecond time scale. Complete regeneration of the dye was achieved within $200 \mu \mathrm{s}$, which is then compatible with the $1 \mathrm{kHz}$ repetition rate of ultrafast transient absorption experiments.

\section{Blends with PCBM}

The morphology of bulk heterojunctions in blended PCBM|Cy3$\mathrm{B}$ films depends on the relative amount of the fullerene acceptor material. This behaviour is qualitatively similar to that of previously described PCBM|Cy7 TRISPHAT blend films. ${ }^{29}$ Films

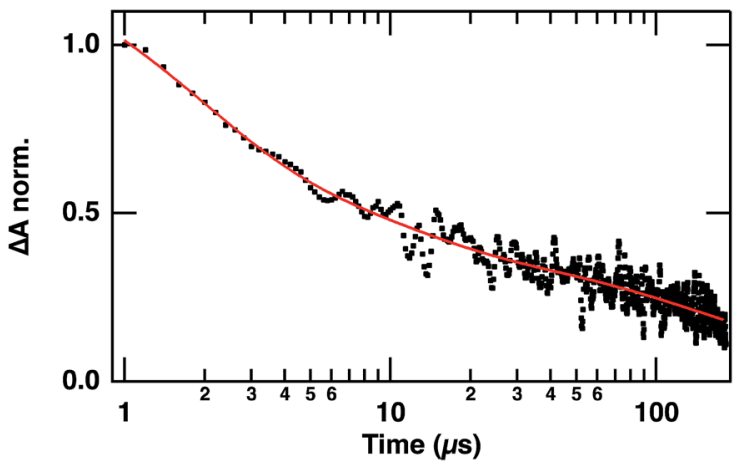

Fig. 4 Dynamics recorded at a $450 \mathrm{~nm}$ probe after $530 \mathrm{~nm}$ excitation for pristine Cy3-B. The solid line is the best fit of experimental data points.

prepared with the largest PCBM loading (15.5:1) display only one fully intermixed phase, while lower PCBM amounts lead to both intermixed and pure cyanine phases segregating at the surface. In the latter case, phase contrast AFM images reveal a spinodal phase pattern at the surface (Fig. S2a $\dagger$ ). Dipping samples into a tetrafluoropropanol solvent selectively dissolves the pure dye phase. The remaining films contain intermixed Cy3-B with PCBM, none of the materials being dissolved anymore. The topography reflects the inverse structure of the dye surface domains (Fig. S2b $\dagger$ ). The various morphologies obtained for different compositions can be explained with an asymmetry in the PCBM|dye|solvent ternary phase diagram and the nature of film formation during a solvent quench (Fig. S3†). ${ }^{29}$ At high PCBM loadings, the system remains in the one phase region (fully intermixed) for all solvent concentrations. For lower PCBM loadings, solvent evaporation brings the system into a two-phase region. It is however unclear how molecular intercalation of PCBM in the cyanine tetraphenylborate ion-pair occurs in the intermixed phase.

The lower the PCBM loading, the larger the pure cyanine layer. The intermixed phase leads to a direct competition between electron transfer to PCBM (eqn (1)) and reductive quenching by the tetraphenylborate counter-anion (eqn (2)) following light absorption by Cy3. As a pure Cy3-B phase exists in all samples, except in the 15.5 : 1 blend, a transient absorption signal corresponding to the $\mathrm{Cy}^{-}$species obtained by reductive quenching is expected. Direct light absorption by PCBM can be neglected for the low content sample $(0.44: 1)$, while blends with a higher fullerene molar ratio show about $20 \%$ light absorption by the sole PCBM material (Fig. 2). The ground-, excited-, and reduced states of PCBM have rather low extinction coefficients and are, therefore, not responsible for the majority of the transient absorption signals observed in the probed spectral range. ${ }^{25}$ Oxidation of Cy3 upon hole transfer from photoexcited PCBM molecules, however, cannot be excluded.

The effect of the addition of PCBM on the dynamics observed at a probe wavelength of $440 \mathrm{~nm}$, where both the Cy3 excited state and reduced species absorb, is shown in Fig. 5a. For all blends, the initial amplitude of the photoabsorption is reduced 


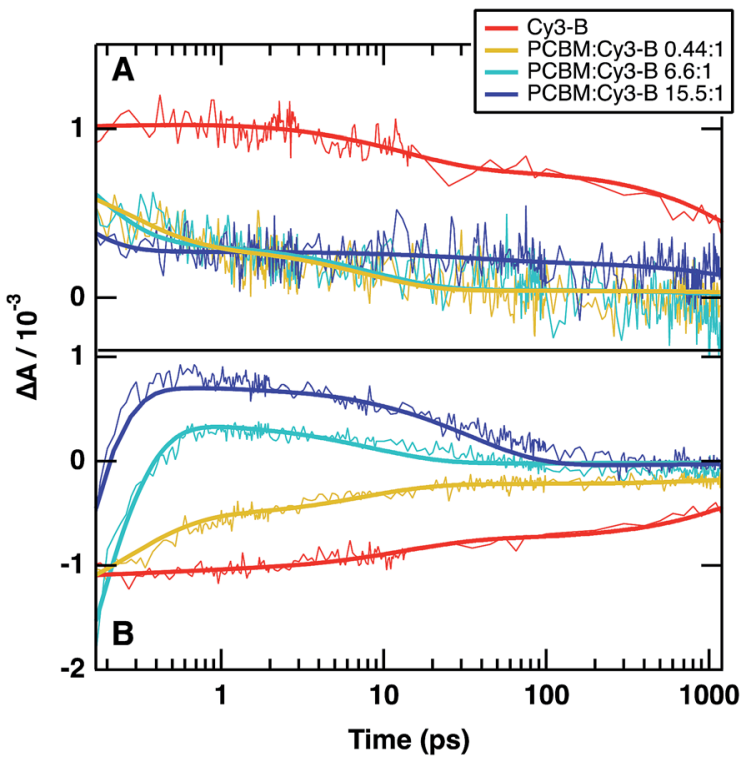

Fig. 5 Dynamics recorded after $530 \mathrm{~nm}$ excitation for pristine Cy3-B and various PCBM loadings for (a) $440 \mathrm{~nm}$ (Cy3 excited and reduced states) and (b) $550 \mathrm{~nm}$ (Cy3 GSB and oxidized state) probe wavelengths. The solid lines represent the result of the best multiexponential fit.

compared to that of the pristine Cy3-B. This suggests that ultrafast quenching of the Cy3* excited state by PCBM takes place, successfully competing with the reductive quenching by tetraphenylborate and inhibiting the formation of $\mathrm{Cy}^{-}{ }^{-}$. The dynamics recorded at $550 \mathrm{~nm}$ shown in Fig. $5 \mathrm{~b}$ are assigned to both the GSB of the cyanine and to the absorption of $\mathrm{Cy}^{+}$ species formed in blends. ${ }^{7}$ At this wavelength a local minimum is observed in the dye ground state absorption spectrum (see Fig. 2), which enables us to scrutinize the formation of the oxidized cyanine species. At high PCBM loadings, photoabsorption of $\mathrm{Cy}^{+}$is directly observed. The amplitude of the positive signal gradually increases with the PCBM content, indicating the production of a higher amount of the oxidized cyanine. The dynamics of cyanine oxidized state formation is extremely fast and could not be entirely resolved with our ultrafast setup. The time constant for $\mathrm{Cy}^{+}$formation yielded by the fitting procedure is, therefore, certainly overestimated. The amplitude values for the high PCBM content blends (6.6:1 and $15.5: 1$ ) can hardly be compared quantitatively as absorption by PCBM cannot be excluded. This explains the similar amplitudes measured at a wavelength of $440 \mathrm{~nm}$ for the $0.44: 1$ and $6.6: 1$ blends (Fig. 5a). Signal amplitudes can be compared only for pristine Cy3-B and the $0.44: 1$ blend.

The fits shown in Fig. 5 result from a global analysis of multiwavelength kinetic data. Fig. 6 presents the associated amplitude spectra (amplitudes associated with the three time constants) for the photophysical processes observed in pristine Cy3-B and blends with PCBM as depicted in Fig. 1. The transient absorption spectra of the blends are shown in Fig. S4, $\dagger$ and again a fluence of $45 \mu \mathrm{J} \mathrm{cm}{ }^{-2}$ was chosen as no intensity effect was observed (Fig. $\mathrm{S} 1 \dagger$ ) and a satisfactory signal-to-noise ratio

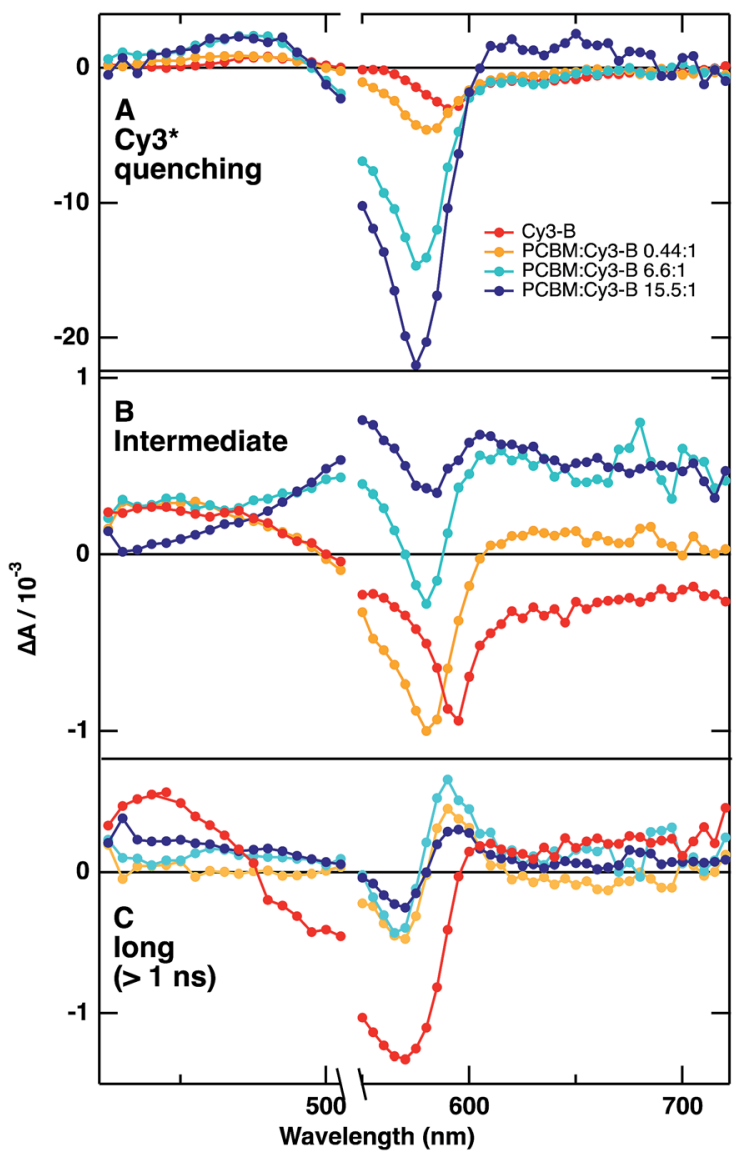

Fig. 6 Associated amplitude spectra resulting from the multiexponential global analysis for various investigated samples. (a) is the excited state quenching, (b) is the intermediate time scale (order of 100 ps) and (c) is the long time scale ( $>1 \mathrm{~ns}$ ).

was obtained. The first two associated time constants for the blends are shown in Table 1, the longer being outside the time window allowed by our femtosecond setup ( $>1 \mathrm{~ns})$. The shortest time constant (Fig. 6a and Table 1) is associated with the Cy3 excited state quenching and the concomitant growth of either the oxidized or the reduced cyanine species absorption upon electron transfer (eqn (1)) or reductive quenching (eqn (2)), respectively. A large negative amplitude located between 500 and $600 \mathrm{~nm}$ is observed for all blends (Fig. 6a), which increases with increasing PCBM relative content and is directly related to the ultrafast formation of the oxidized cyanine species. When PCBM is added, the SE gradually disappears (Fig. S4 $\dagger$ ), but Cy3 ESA spanning over a wide range of wavelengths $(420-500 \mathrm{~nm})$ is still observed at early time delays. This indicates that ultrafast electron transfer to PCBM occurs from the cyanine emissive photoexcited state. For pure Cy3-B in either pristine Cy3-B dye films or in the segregated phase from blends, $\mathrm{Cy}^{-}$absorption around 420-470 nm starts growing on a similar time scale. As a result, no decaying amplitude associated with the first time scale is observed at the blue side of the ESA. The intermediate time scale, shown in Fig. 6b, reveals the presence of reduced dye species for all samples, except the one containing the highest PCBM load (15.5: 1). This is an expected result, as this is the 
Table 1 Time constants for Cy3* excited state quenching and approximate lifetimes (order of magnitude) of oxidized and reduced forms of the dye in films of various compositions

\begin{tabular}{|c|c|c|}
\hline Film composition & Cy3* quenching time constants & $\mathrm{Cy} 3^{-}$and $\mathrm{Cy}^{+}{ }^{+}$lifetime \\
\hline Pristine Cy3-B & $\begin{array}{l}462.1 \pm 17.7 \text { fs }(52 \%) \\
11.7 \pm 1.4 \text { ps }(48 \%)\end{array}$ & $\mathrm{Cy}^{-}: 10^{-4} \mathrm{~s}$ \\
\hline
\end{tabular}

only sample where both Cy3-B and PCBM are fully intermixed, thus resulting in a fast electron transfer to the fullerene competing successfully against the reductive quenching by $\mathrm{BPh}_{4}$. The oxidized state's lifetime is associated with the intermediate time constant (Fig. 6b, picoseconds), whereas the reduced species live for several microseconds (Fig. 4, 6c and Table 1).

In solution, reductive quenching of the $\mathrm{C}_{60}$ triplet photoexcited state by a tetraphenylborate anion has been observed to yield the $\mathrm{C}_{60}$ anion. ${ }^{26}$ The PCBM anion absorbs around $1030 \mathrm{~nm}$, outside the spectral range covered by our setup. ${ }^{30}$ Moreover, intersystem crossing to the triplet state of PCBM is rather improbable in thin films, as other deactivation pathways become possible due to the close packing of fullerene molecules. ${ }^{31}$

The positive amplitude of the absorption change observed at longer wavelengths for 15.5:1 and 6.6:1 blends (Fig. 6 and $\mathrm{S} 4 \dagger$ ) could be due to PCBM ESA, although fullerene molecules absorb only $20 \%$ of the incoming light. We are currently investigating the origin of this effect. Among various hypotheses, we could speculate that this photoabsorption originates from charge transfer states formed between cyanine and PCBM neighboring molecules. ${ }^{32}$ The photoinduced Stark effect is excluded here, as the Stark shift observed elsewhere for Cy3-P is quite weak, and the one arising from PCBM typically displays narrow features, which do not appear here. ${ }^{5,33}$

While the yield of electron transfer from $\mathrm{Cy} 3 *$ to the fullerene clearly increases with the acceptor's concentration (Fig. 5b), its rate also appears to depend upon the PCBM molar ratio. Time constants measured for the cyanine excited state quenching are reported in Table 1 . From the results obtained with the pure Cy3-B sample, we can conclude that the reductive quenching happens in a few picoseconds ( 0.46 and 11.7 ps time constants). In the intermixed phase of blends, $\mathrm{Cy}^{+}$species appear on time scales decreasing with the average distance separating Cy3 and PCBM molecules, reaching 77.5 fs for the 15.5: 1 blend. As explained previously in the text, the fit obtained for this sample probably overestimates the time constant (Fig. 5b), whose actual value must probably be shorter than 50 fs. The cyanine oxidized state lifetime does not exceed a few tenths of picoseconds as evidenced in Fig. 5b. Fig. 6c showing the long time component corroborates this as no transient signal assigned to $\mathrm{Cy}^{+}$around $550 \mathrm{~nm}$ is observed. Hole transfer from photoexcited PCBM in the $6.6: 1$ and $15.5: 1$ blends is not ruled out, and could also be responsible for a faster appearance of the Cy3 oxidized state. The $0.44: 1$ blend still shows a faster excited state quenching than in pristine Cy3$\mathrm{B}$, whereas in this sample the absorption by PCBM is negligible. It is therefore clear that electron transfer from photoexcited Cy3 to PCBM is faster than the reductive quenching by tetraphenylborate, and that hole injection from photoexcited PCBM to Cy3 could be even faster.

It is likely that reduction of $\mathrm{Cy}^{+}$species obtained upon electron transfer to PCBM by the tetraphenylborate counteranion is also responsible for the decay of the Cy3 oxidized species absorption. We expect that applying a voltage to the blend solar cell devices will prevent the unwanted charge recombination from the PCBM anion. The effect of a voltage bias will be treated in a separate paper. ${ }^{33}$

Cy3 isomerization is assumed to be responsible for the positive transient absorption feature observed at $600 \mathrm{~nm}$ on long time scales in blends only (Fig. 6c). It is not observed in pristine Cy3-B, which leads us to think that the isomerization, which is a rather slow process (ns), occurs in the intermixed phase. Either the ion pair with tetraphenylborate is too closely packed, resulting in inevitable reductive quenching upon light absorption by Cy3 in pure Cy3-B layers, or isomerization occurs from the cyanine oxidized species. Further investigation of the isomerization of cyanine dyes will be addressed in a future study.

\section{Conclusions}

In summary, we have shown that cyanine tetraphenylborate Cy3-B undergoes intra-ion pair reductive quenching in the solid state. The picosecond time scale for electron transfer from the tetraphenylborate moiety to the excited Cy3 suggested from the global analysis is in accordance with previous findings in solution. ${ }^{19,20}$ No evidence for tetraphenylborate radicals due to carbon-boron bond cleavage has been noticed as the cyanine reduced species absorbing at 420-450 $\mathrm{nm}$ recombines with the oxidized tetraphenylborate on the microsecond time scale. The widely used PCBM electron acceptor was blended with Cy3-B with different ratios and we found that the phase morphology drives charge injection in PCBM. High PCBM loadings consist of a fully intermixed phase, where the appearance of $\mathrm{Cy}^{+}$ oxidized states takes place readily after the excitation laser pulse (time constant $<160$ fs). Low PCBM loadings lead to an intermixed phase and a pure Cy3-B segregated phase, therefore enabling the observation of both reduced and oxidized Cy3 species. The $\mathrm{Cy}^{+}{ }^{+}$oxidized species produced in the intermixed 
phase recombines on the hundred-picosecond timescale. Overall, observation of the cyanine species in either the oxidized or the reduced form, resulting from kinetic competition between electron transfer to PCBM or intra-ion-pair reductive quenching, is a sensitive indicator of the degree of intermixing of different phases. A better understanding of the dynamics of charge transfer in bulk heterojunctions provides ways to establish clear structure-property relationships that will enable precise control of the optoelectronic properties in OPV materials by targeting optimized morphologies.

\section{Experimental part}

\section{Materials}

The cyanine dye 1,1'-diethyl-3,3,3',3'-tetramethylcarbocyanine tetraphenylborate (Cy3-B) was purchased from FEW chemicals, Germany. [6,6]-Phenyl- $\mathrm{C}_{61}$-butyric acid methyl ester (PCBM) was purchased from Solenne B.V., Netherlands. Both Cy3-B and PCBM were dissolved in various mole percent concentrations in chlorobenzene (Sigma-Aldrich). The films were fabricated by spin coating the mixed solutions onto glass substrates. All the samples were prepared under nitrogen in a glove box. All chemicals were used as supplied, without further purification.

\section{Spectroscopy}

Absorption spectra were recorded on a Perkin-Elmer Lambda 950 spectrophotometer. Spectra were baseline-corrected and measured against a reference.

Transient absorption spectra were recorded with a femtosecond pump-probe spectrometer based on an amplified Tisapphire laser (Clark-MXR, CPA-2001) delivering $778 \mathrm{~nm}$ pulses of $150 \mathrm{fs}$ duration at a $1 \mathrm{kHz}$ repetition rate. The pump beam was generated by a two-stage non-collinear optical parametric amplifier (NOPA), while the probe beam was a broadband white light continuum (420-720 $\mathrm{nm}$ ) generated by a portion of the 778 $\mathrm{nm}$ fundamental output of the laser passing through a sapphire plate. The pump wavelength was set at $530 \mathrm{~nm}$ and the fluence at the sample was limited to $45 \mu \mathrm{J} \mathrm{cm}^{-2}$. The probe beam was split before the sample into signals and reference beams in order to account for intensity fluctuations. Both beams were recorded shot by shot with a pair of $163 \mathrm{~mm}$ spectrographs (Andor Technology, SR163) equipped with $512 \times 58$ pixels backthinned CCD cameras (Hamamatsu S07030-0906). The transient spectra were recorded by averaging over 3000 shots. The polarization of pump and probe pulses was set at a magic angle. All time-dependent spectra were corrected for white-light chirp measured by Kerr gating and background noise. Global analysis by a multiexponential fit at different wavelengths (typically every $5 \mathrm{~nm}$ ) allowed for unraveling the various occurring photophysical processes. The associated spectra show the amplitude of the transient signal related to each time component. The global analysis procedure is detailed in the ESI. $\uparrow$ The samples were analyzed in a dry argon atmosphere in a home-built chamber avoiding contact with ambient air.

Microsecond timescale dynamics were recorded using a frequency-tripled Q-switched Nd:YAG laser (Ekspla NT-342) running at a $20 \mathrm{~Hz}$ repetition rate. An optical parametric oscillator was used to generate $530 \mathrm{~nm}$ wavelength pump pulses (5 ns FWHM). The continuous-wave probe light from a xenon lamp was transmitted through the sample, various optics, and a grating monochromator before being detected using a fast photomultiplier tube. Transient signals were recorded using a digital oscilloscope (Tektronix DPO 7104C). Averaging over typically 3000 shots yielded a satisfactory signal-to-noise ratio. A second-order Savitzky-Golay smoothing algorithm was applied (35 points intervals).

\section{Acknowledgements}

Financial support by NCCR-MUST and the Swiss National Science Foundation is gratefully acknowledged.

\section{Notes and references}

1 B. Walker, C. Kim and T.-Q. Nguyen, Chem. Mater., 2011, 23, 470-482.

2 Y. Sun, G. C. Welch, W. L. Leong, C. J. Takacs, G. C. Bazan and A. J. Heeger, Nat. Mater., 2011, 11, 44-48.

3 Y. Liu, C.-C. Chen, Z. Hong, J. Gao, Y. M. Yang, H. Zhou, L. Dou, G. Li and Y. Yang, Sci. Rep., 2013, 3, 3356.

4 S. Shoaee, S. Mehraeen, J. G. Labram, J.-L. Brédas, D. D. C. Bradley, V. Coropceanu, T. D. Anthopoulos and J. R. Durrant, J. Phys. Chem. Lett., 2014, 5, 3669-3676.

5 A. Devižis, D. Hertel, K. Meerholz, V. Gulbinas and J. E. Moser, Org. Electron., 2014, 15, 3729-3734.

6 J. Heier, J. Groenewold, S. Huber, F. Nüesch and R. Hany, Langmuir, 2008, 24, 7316-7322.

7 B. Fan, F. Araujo De Castro, J. Heier, R. Hany and F. Nüesch, Org. Electron., 2010, 11, 583-588.

8 G. Wicht, S. Bücheler, M. Dietrich, T. Jäger, F. Nüesch, T. Offermans, J.-N. Tisserant, L. Wang, H. Zhang and R. Hany, Sol. Energy Mater. Sol. Cells, 2013, 117, 585-591.

9 S. Jenatsch, R. Hany, A. C. Véron, M. Neukom, S. Züfle, A. Borgschulte, B. Ruhstaller and F. A. Nüesch, J. Phys. Chem. C, 2014, 118, 17036-17045.

10 O. Malinkiewicz, T. Grancha, A. Molina-Ontoria, A. Soriano, H. Brine and H. J. Bolink, Adv. Energy Mater., 2012, 3, 472477.

11 A. Mishra, R. K. Behera, P. K. Behera, B. K. Mishra and G. B. Behera, Chem. Rev., 2000, 100, 1973-2012.

12 M. Lloyd and J. Anthony, Mater. Today, 2007, 10, 34-41.

13 H. Benmansour, F. A. Castro, M. Nagel, J. Heier, R. Hany and F. Nüesch, Chimia, 2007, 61, 787-791.

14 A. C. Véron, H. Zhang, A. Linden, F. Nüesch, J. Heier, R. Hany and T. Geiger, Org. Lett., 2014, 16, 1044-1047.

15 Z. Huang, D. Ji, S. Wang, A. Xia, F. Koberling, M. Patting and R. Erdmann, J. Phys. Chem. A, 2006, 110, 45-50.

16 K. Jia, Y. Wan, A. Xia, S. Li, F. Gong and G. Yang, J. Phys. Chem. A, 2007, 111, 1593-1597.

17 A. Chibisov and S. Shvedov, J. Photochem. Photobiol., 2001, 141, 39-45. 
18 L. F. Vieira Ferreira, A. S. Oliveira, F. Wilkinson and D. Worrall, J. Chem. Soc., Faraday Trans., 1996, 92, 12171225.

19 F. A. Castro, H. Benmansour, J.-E. Moser, C. F. O. Graeff, F. Nüesch and R. Hany, Phys. Chem. Chem. Phys., 2009, 11, 8886-8894.

20 M. C. Etter, B. N. Holmes, R. B. Kress and G. Filipoich, Isr. J. Chem., 1984, 25, 264-273.

21 G. B. Schuster, Pure Appl. Chem., 1990, 62, 1565-1572.

22 B. Sauerwein and G. B. Schuster, J. Phys. Chem., 1991, 95, 1903-1906.

23 X. Yang, A. Zaitsev, B. Sauerwein, S. Murphy and G. B. Schuster, J. Am. Chem. Soc., 1992, 114, 793-794.

24 S. Chatterjee, P. Gottschalk, P. D. Davis and G. B. Schuster, J. Am. Chem. Soc., 1988, 110, 2326-2328.

25 S. Chatterjee, P. D. Davis, P. Gottschalk, M. E. Kurz, B. Sauerwein, X. Yang and G. B. Schuster, J. Am. Chem. Soc., 1990, 112, 6329-6338.

26 T. Konishi, Y. Sasaki, M. Fujitsuka, Y. Toba, H. Moriyama and O. Ito, J. Chem. Soc., Perkin Trans. 2, 1999, 551-556.
27 J. D. Wilkey and G. B. Schuster, J. Org. Chem., 1987, 52, 21172122.

28 S. Chatterjee, P. D. Davis, P. Gottschalk, M. E. Kurz, B. Sauerwein, X. Yang and G. B. Schuster, J. Am. Chem. Soc., 1990, 112, 6329-6338.

29 J. Heier, C. Peng, A. C. Véron, R. Hany, T. Geiger, F. A. Nüesch, M. V. G. Vismara and C. F. O. Graeff, SPIE, ed. Z. H. Kafafi, P. A. Lane and I. D. W. Samuel, 2014, vol. 9184, p. 918408.

30 J. Guo, J. Guo, H. Ohkita, H. Ohkita, H. Benten, H. Benten, S. Ito and S. Ito, J. Am. Chem. Soc., 2010, 132, 6154-6164.

31 S. Cook, H. Ohkita, Y. Kim and J. Benson-Smith, Chem. Phys. Lett., 2007, 445, 276-280.

32 D. Peckus, A. Devižis, R. Augulis, S. Graf, D. Hertel, K. Meerholz and V. Gulbinas, J. Phys. Chem. C, 2013, 117, 6039-6048.

33 A. Devižis, J. De Jonghe-Risse, R. Hany, F. Nüesch, S. Jenatsch, V. Gulbinas, and J.-E. Moser, 2015, submitted. 